\title{
Genome Editing: Innovation in Molecular Biology
}

\section{Nida Tabassum Khan*}

Faculty of Life Sciences and Informatics, Department of Biotechnology, Balochistan University of Information Technology Engineering and Management Sciences, (BUITEMS), Quetta, Pakistan

\begin{abstract}
Deletion, insertion, or substitution of DNA at a precise location in the genome of an organism is termed as Genome editing. It is usually accomplished in vitro using genetically engineered restriction enzymes endonucleases also called as molecular scissors. There is a numerous way to edit genome using different genome editing systems including CRISPR-Cas9, ZFNs or TALENs. Each of these systems possesses unique properties that are exploited for an organism benefits.
\end{abstract}

\section{Keywords: Nucleases; CRISPR-Cas9; ZFNs}

\section{Introduction}

Genome editing is a novel approach that is gaining momentum by making precise and unique edits in the existing genetic code of a living organism. This innovating molecular technique enables addition, deletion and substitution of bases by incorporating detectable changes in the DNA of an organism [1]. In comparison with classical genetic engineering which is the cleavage and random insertion of a foreign gene or DNA sequence from a different specie to another. Genome editing technology holds a different concept. It edits the DNA or genes at much defined localities in the genome [2].

\section{Genome Editing Working Principle}

The core concept behind genome editing is the use of restriction nucleases that make explicit cuts in the target DNA by recognition of specific restriction sites also called as recognition sites within the target sequence or gene. The cleavage location is then repaired by DNA repair system of the cell. These repairs can be tricked to add, remove, or substitute a series of letters in the genetic code [3]. Thus enables the introduction of known desired alleles in the target organism. Breaks in the double stranded DNA produced at exact position in the DNA by restriction endonucleases are mended by DNA repair pathway of the cell without template DNA. Outcome of this modification is a change in a single nucleotide either by insertion or deletion at the DNA cut site. Though the position of the cleaved site is precise but the exact alteration that occurs when the DNA is repaired is random. So there might be a number of different possibilities producing minor sequence changes [3].

\section{Types of genome editing}

Small DNA changes: Engineered endo nuclease designed to cleave at specific localities in the DNA. DNA segment with the engineered nuclease after cleavage will be recognized by the cells natural repair system that will ligase the two cleaved ends together. This repair mechanism is not $100 \%$ efficient resulting in the loss or gain of few nucleotide bases during the repair process. A small change in the nucleotide sequence will result in the alteration of the function of that segment of DNA. As a result a gene may either become nonfunctional or doesn't function properly $[4,5]$.

Removal of a section of DNA: Endonucleases are designed to make cuts on either side of the DNA to remove a segment. After cleavage, cells normal DNA repair mechanism will identify the damage and initiate the repair process but may mistakenly join the wrong ends of DNA together, removing the DNA in between the two cuts [6].

Insertion of section of DNA: Genome editing could be used to direct the cells natural DNA repair system to insert a segment of
DNA into a genome. Before cell division, cell copies all of its DNA so a complete copy of the genome is transferred to the two resultant daughter cells. Since DNA repair is a homology directed repair process [7]. Designed endonucleases cleave at precise location in the DNA. After cleavage, a DNA segment homologous in sequence to the site of the cleavage is introduced [8]. The break is repaired by using this modified DNA segment as template. As a result a new DNA segment is inserted or an existing segment of DNA is substituted by an altered one [9].

\section{Genome editing systems}

There are numerous types of engineered restriction nuclease systems used in genome editing. They all contain a nuclease part that cleaves the DNA and a DNA-targeting part to identify the DNA sequence that has been cleaved.

CRISPR-Cas9: CRISPR-Cas9 is the mostly used cost effective genome editing system. These clustered regularly interspaced short palindromic repeats targets DNA. It comprised of a guide RNA molecule that binds to specific DNA bases through complementary base pairing. Cas9 is CRISPR-associated protein 9, nuclease part that cleaves the DNA. This system was originally reported in bacteria that uses it to destroy invading viruses $[9,10]$.

ZFNs: Zinc-finger nucleases (ZFNs) are another type of nucleases in which zinc-finger proteins is the DNA-binding part of ZFNs which bind to about three DNA bases [11,12]. FokI is the nuclease part of ZFNs which cleaves the DNA [13]. Two FokI molecules collaborate together to make a cut in the target DNA molecule so it works in pairs.

TALENs: In transcription activator-like effector nucleases, DNAbinding domain is made of TALE (transcription activator-like effector domains) $[14,15]$. There are four different TALE domains for four different nucleotide bases, so they can be engineered to bind to precise DNA regions with much ease than ZFNs. FokI is the nuclease part of TALENs. Two FokI molecules must come together to make a cleavage in the DNA, so two TALENs are made, one for each strand $[16,17]$.

*Corresponding author: Nida Tabassum Khan, Faculty of Life Sciences and Informatics, Department of Biotechnology, Balochistan University of Information Technology Engineering and Management Sciences, (BUITEMS), Quetta, Pakistan, Tel: 03368164903; E-mail: nidatabassumkhan@yahoo.com

Received June 30 2017; Accepted July 10, 2017; Published July 20, 2017

Citation: Khan NT (2017) Genome Editing: Innovation in Molecular Biology. Hereditary Genet 6: 185. doi:10.4172/2161-1041.1000185

Copyright: (c) 2017 Khan NT. This is an open-access article distributed under the terms of the Creative Commons Attribution License, which permits unrestricted use, distribution, and reproduction in any medium, provided the original author and source are credited. 


\section{Applications of genome editing}

Genome editing has been used for a variety of purposes such as for the development of human disease models, understanding complex biological pathway etc., it has infinite range of promising applications.

Biomedical research studies: Genome editing is employed to alter the DNA in organisms to have an insight of the fundamental biological pathways and its underlying mechanism [18].

Diseases treatment: Genome editing has been used to cure different genetic diseases such as in vitro modification of human blood cells that are then introduced into the body to treat diseases like leukemia and AIDS [19]. Besides, genetic diseases including muscular dystrophy and hemophilia could also be treated [20,21].

Agricultural biotechnology: Genetic modification of food crops has been done through the use of genome editing technology to make them disease resistance as well to increase their yield. For example, CRISPR-Cas9 and TALENs editing systems have been used to create modification in the multiple alleles within hexaploid bread wheat to make them resistant against powdery mildew. And such genomic modification is heritable. It was reported that by using TALENs, genes in the fatty acid metabolic pathway in soybean were knocked out to create a modified plant with few metabolic components etc. [22,23].

Crops and livestock: Increased product yield, disease resistance potential, and abiotic tolerance, was achieved in different plants and animals by employing genome editing technology [24].

Industrial biotechnology: Production of biopharmaceuticals, biofuels, biodegradable plastics, biomaterials etc is the recent applications of genome editing.

Biomedicine: Nanoparticles mediated drug delivery systems, xenotransplantation, gene therapies, control and prevention of genetic diseases [25].

Reproduction: Preventing the inheritance of a disease trait [26].

Animal breeding: Genome editing is used to create genetically modified animals with improved phenotypes by modifying a less desirable allele rather than by means of conventional selective breeding. Therefore this cost effective method is more like precision breeding where breeders can introduce the specific sequences that they would like to select $[27,28]$.

\section{Targeting efficiency and of incidence of off-target mutations of different genome editing systems}

Efficiency of genome targeting and the proportion of anticipated transformation achieved, is one of the most significant considerations to assess the importance of this tool. Targeting efficiency of CRISPERCas9 is more than ZFNs and TALENs [1]. It was reported that targeting efficiency of TALENs and ZFNs in human cells lie from $1 \%$ to $50 \%$ [29-31]. However an efficiency percentage of $>70 \%$ was reported in zebrafish [32] and plants [33] while $2 \%$ to $5 \%$ was achieved in stem cells [34] using RISPER Cas9. Improved germline transmission using dual sgRNAs could increase the efficiency of genome targeting up to $78 \%$, for example in single celled embryo of mouse [35]. Frequency of off-target mutations is an important parameter that usually occurs at sites that have little nucleotide difference in comparison to the original sequence localized adjacent to PAM sequence. A single nucleotide change in the PAM sequence or a five nucleotide mismatches within the protospacer region can easily be tolerated by CRISPER Cas9. Detection of off-target mutations is not that easy but it requires sequencing of whole-genome to eliminate them. Recently CRISPER Cas9 editing system has been modified to reduce the probability of off-target mutations by either two extra guanine nucleotides addition to the $5^{\prime}$ end or by employing condensed gRNA (condensed within the crRNA-derived sequence). Another approach is the use of "paired nickases "that employed D10A Cas9 and two sgRNAs complementary to the adjacent area in the target site. Thus, only a single nick is produces in off-target locations and, therefore, results in insignificant off-target mutations [36].

\section{Conclusion}

Genome editing technology is an innovation in the field of molecular biology. Designer nucleases is responsible for its high efficiency and versatility enabling precise genomic modification in the target. Thus, genome editing holds a promising future in the field of genome engineering.

\section{References}

1. Hilton IB, Gersbach CA (2015) Enabling functional genomics with genome engineering. Genome Res 25: 1442-1455.

2. Urnov FD, Rebar EJ, Holmes MC, Zhang HS, Gregory PD (2010) Genome editing with engineered zinc Finger nucleases. Nat Rev Genet 11: 636-646

3. Hwang WY, Fu Y, Reyon D, Maeder ML, Kaini P, (2013) Heritable and precise zebrafish genome editing using a CRISPR-Cas system. PLoS One 8: e68708.

4. Porteus $\mathrm{MH}$ (2015) Towards a new era in medicine: Therapeutic genome editing. Genome Biol 16: 286

5. Feng Z, Zhang B, Ding W, Liu X, Yang DL (2013) Efficient genome editing in plants using a CRISPR/Cas system Cell Res. 23: 1229-1232.

6. Mali P, Yang L, Esvelt KM, Aach J, Guell M, et al. (2013) RNA-guided human genome engineering via Cas9 Science, 339: 823-826.

7. Zhou, J, Wang J, Shen B, Chen L, Su Y, et al. (2014) Dual sgRNAs facilitate CRISPR/Cas9-mediated mouse genome targeting. FEBS J 281: 1717-1725.

8. Fu, Y, Foden JA, Khayter C, Maeder ML, Reyon D, et al. (2013) High-frequency off-target mutagenesis induced by CRISPR-Cas nucleases in human cells Nat Biotechnol 31: 822-826.

9. Fu, Y, Sander JD, Reyon D, Cascio VM, Joung JK, et al. (2014) Improving CRISPR-Cas nuclease specificity using truncated guide RNAs. Nat Biotechnol 32: $279-284$

10. Kim E, Kim S, Kim DH, Choi BS, Choi IY, et al. 2012 Precision genome engineering with programmable DNA-nicking enzymes. Genome Res 22 1327-1333.

11. Miller JC, Tan S, Qiao G, Barlow KA, Wang J, et al. 2011 A TALE nuclease architecture for efficient genome editing. Nat Biotechnol 29: 143-148.

12. Heigwer F, Kerr G, Boutros M (2014) E-CRISP: Fast CRISPR target site identification. Nat Methods 11: 122-123.

13. Pavletich NP, Pabo CO (1991) Zinc finger-DNA recognition: Crystal structure of a Zif268-DNA complex at 2.1 A. Science 252: 809-817.

14. Kim YG, Cha J, Chandrasegaran S (1996) Hybrid restriction enzymes: Zinc finger fusions to Fok I cleavage domain. Proc Natl Acad Sci 93: 1156-1160.

15. Christian M, Cermak T, Doyle EL, Schmidt C, Zhang F, et al. (2010) Targeting DNA double-strand breaks with TAL effector nucleases. Genetics 186: 757-761.

16. Miller JC, Tan S, Qiao G, Barlow KA, Wang J, et al. (2011) A TALE nuclease architecture for efficient genome editing. Nat Biotechnol 29: 143-148.

17. Bibikova M, Beumer K, Trautman JK, Carroll D (2003) Enhancing gene targeting with designed zinc finger nucleases. Science 300: 764.

18. Cox DB, Platt RJ, Zhang F (2015) Therapeutic genome editing: Prospects and challenges. Nat Med 21: 121-131.

19. Perez EE, Wang J, Miller JC, Jouvenot Y, Kim KA, et al (2008) Establishment of HIV-1 resistance in CD4+ T cells by genome editing using zinc-finger nucleases. Nat Biotechnol 26: 808-816.

20. Boissel S, Jarjour J, Astrakhan A, Adey A, Gouble A, et al. (2014) MegaTALs: A rare-cleaving nuclease architecture for therapeutic genome engineering. Nucleic Acids Res 42: 2591-2601. 
21. Long C, Amoasii L, Mireault AA, McAnally JR, Li H, et al. (2015) Postnatal genome editing partially restores dystrophin expression in a mouse model of muscular dystrophy. Science 351: 400-403.

22. Baltes NJ, Voytas DF (2015) Enabling plant synthetic biology through genome engineering. Trends Biotechnol 33: 120-131.

23. Haun W, Coffman A, Clasen BM, Demorest ZL, Lowy A, et al. (2014) Improved soybean oil quality by targeted mutagenesis of the fatty acid desaturase 2 gene family. Plant Biotechnol J 12: 934-940.

24. Maeder ML, Gersbach CA (2016) Genome-editing technologies for gene and cell therapy. Mol Ther 24: 430-446

25. Blancafort P, Tschan MP, Bergquist S, Guthy D, Brachat A, et al. (2008) Modulation of drug resistance by artificial transcription factors. Mol Cancer Ther 7: 688-697.

26. Hammond A, Galizi R, Kyrou K, Simoni A, Siniscalchi C, et al. (2016) A CRISPRCas9 gene drive system targeting female reproduction in the malaria mosquito vector Anopheles gambiae. Nat Biotechnol 34: 78-83.

27. Carlson DF, Tan W, Lillico SG, Stverakova D, Proudfoot C, et al. (2012) Efficient TALEN-mediated gene knockout in livestock. Proc Natl Acad Sci 109: 1738217387.

28. Santiago Y, Chan E, Liu PQ, Orlando S, Zhang L, et al. (2008) Targeted gene knockout in mammalian cells by using engineered zinc-finger nucleases. Proc Natl Acad Sci 105: 5809-5814.
29. Mussolino, C, Morbitzer R, Lütge F, Dannemann N, Lahaye T, et al. (2011) A novel TALE nuclease scaffold enables high genome editing activity in combination with low toxicity. Nucleic Acids Res 39, 9283-9293.

30. Miller JC, Tan S, Qiao G, Wang J, Paschon DE, et al. (2011) A TALE nuclease architecture for efficient genome editing. Nat Biotechnol 29: 143-148.

31. Maeder ML, Thibodeau-Beganny S, Osiak A, Jiang T, Foley JE, et al. (2008) Rapid "open-source" engineering of customized zinc-finger nucleases for highly efficient gene modification. Mol Cell 31: 294-301.

32. Hwang WY, Fu Y, Reyon D Sander DJ, Peterson TR, et al. (2013) Heritable and precise zebrafish genome editing using a CRISPR-Cas system. PLoS One 8: e68708.

33. Feng, Z, Zhang B, Ding W, Liu X, Wei P, et al. (2013) Efficient genome editing in plants using a CRISPR/Cas system. Cell Res. 23: 1229-1232.

34. Mali, P, Yang L, Esvelt KM, Aach J, Guell M, et al. (2013) RNA-guided human genome engineering via Cas9. Science 339: 823-826.

35. Hsu PD, Scott DA, Weinstein JA, Ran FA, Konermann S, et al. (2013) DNA targeting specificity of RNA-guided Cas9 nucleases. Nat Biotechnol 31: 827-832.

36. Sander JD, Zaback P, Joung JK, Voytas DF, Dobbs D, et al. (2007) Zinc Finger Targeter (ZiFiT): An engineered zinc finger/target site design tool. Nucleic Acids Res. 35: W599-605. 